\title{
Biometrie in neuem Licht?
}

Vor 22 Jahren fand in der Bonner Bundesrepublik die letzte Volkszählung statt. Die ursprünglich für 1991 geplante erneute gesamtdeutsche Zählung hat bis heute nicht stattgefunden - die Bundesregierung plant sie nun für 2011. Was man damals den Zählenden nicht anvertrauen wollte, wird heutzutage - meist ohne Not und ohne Nachdenken - in Internet-Foren von der Art XING, Linkedin, StudiVZ etc. verbreitet und verbleibt dauerhaft dort. Der Traum von den selbstlöschenden peinlichen Bildern einer jugendlichen Trink-Party ist aus technischer Sicht unrealistisch. Daten und Bilder sind in aller Regel persistent im Internet verfügbar. Auch der fahrlässige Umgang mit Bonus-Systemen ist nachvollziehbar; vielfältig werden nur scheinbar existierende finanzielle Vorteile von Bonus-Karten genutzt und im gleichen Zuge Personendaten in großem Umfang und ohne Zweckbindung preisgegeben.

Die Einstellung und der Umgang der Bürger mit den eigenen persönlichen Daten hat sich seit den 80er Jahren geändert und auf das Internet als neue, soziale Umgebung' eingestellt. Daran hat auch das permanente Unbehagen über mögliche zentrale Datenspeicherungen und -zusammenführungen nichts geändert. Andererseits wird die Notwendigkeit einer zweckbestimmten rationellen elektronischen Verwaltung von Identitäten zunehmend sichtbar. So haben die Bundesbürger im Herbst 2008 zum Beispiel einen persönlichen Zustellbrief der Finanzbehörden mit einer Identifikationsnummer nach $\$ 139 b$ der Abgabenordnung erhalten. Wie weit ist diese eindeutige Identifikationsnummer von einer Personenkennziffer entfernt?

Und dann auch noch die biometrischen Verfahren! Bedeuten die biometrischen Daten nicht eine weitere faktische Personenkennziffer, die eine Verknüpfung zwischen unterschiedlichen Anwendungen und das Aufdecken von bis dahin unbekannten Beziehungen erst ermöglicht? Diese Sorge treibt viele Bürger um und mündete letztlich 2007 in der Entscheidung des Gesetzgebers, im Passgesetz keine zentrale Speicherung von biometrischen Daten der Passantragsteller vorzunehmen. Die meisten Mitgliedsländer der EU kamen zu einem anderen Ergebnis. Ob dadurch die Bundesrepublik zu einem begehrten Ort zum Erschleichen einer zweiten (anderen?) Pass-Identität wird, bleibt abzuwarten. Ein Abgleich der biometrischen Daten mit den bereits ausgestellten Personaldokumenten wird es auf absehbare Zeit bei uns nicht geben.

Das aktuelle DuD-Heft widmet sich dem Schwerpunktthema Biometrie und berichtet von hoheitlichen Anwendungen der Personaldokumente und dem Weg zu einem Europäischen Visainformationssystem. Einen wichtigen Schwerpunkt des Heftes bilden darüber hinaus die Berichte zu den Schutzmöglichkeiten von biometrischen Referenzdaten (Template Protection). Es wird deutlich, dass Biometrie und Datenschutz vereinbar sind. Mit den aktuellen Ergebnissen der biometrischen Template Protection Verfahren wird es möglich, biometrische Referenzdatensätze zu erzeugen, die nicht mehr zum Ausgangssignal invertiert werden können. Die Referenz bleibt für die betroffene Person eindeutig innerhalb einer Anwendung, wird jedoch durch eine andere Wahl von Parametern disjunkt für eine andere Anwendung. Das biometrische Referenzdatum gewinnt dadurch an Datenschutzkonformität: Ein nicht-delegierbarer Authentisierungsfaktor, der dennoch keine ungewollten Querbezüge herstellt. Der Gewinn für den Datenschutz ist enorm!

\section{Christoph Busch und Helmut Reimer}

